\title{
Straightforward synthesis of hyperbranched polymer/graphene nanocomposites from graphite oxide via in situ grafting from approach
}

\author{
QIUHONG XU ${ }^{1}$, YIWEN GONG ${ }^{1}$, YUAN FANG ${ }^{2}$, GUOHUA JIANG ${ }^{1,3, *}$, YIN WANG ${ }^{1,3}$, \\ XINKE SUN ${ }^{1,3}$ and RIJING WANG ${ }^{1,3}$ \\ ${ }^{1}$ Department of Materials Engineering, College of Materials and Textile, Zhejiang Sci-Tech University, Hangzhou 310018, \\ P. R. China \\ ${ }^{2}$ Department of Textile Engineering, College of Materials and Textile, Zhejiang Sci-Tech University, Hangzhou 310018, \\ P. R. China \\ ${ }^{3}$ Key Laboratory of Advanced Textile Materials and Manufacturing Technology (ATMT), Ministry of Education, \\ Zhejiang Sci-Tech University, Hangzhou 310018, P. R. China
}

MS received 25 June 2011; revised 10 July 2011

\begin{abstract}
The grafting of graphite oxide (GO) with cyclic ether monomers, directly affords grafting with hyperbranched polymers. The resulting nanocomposites show good solubility in the solvents of polymers, exfoliation of graphene in the polymer matrix and excellent mechanical properties and robustness under bending.
\end{abstract}

Keywords. Nanostructures; composite materials; atomic force microscopy (AFM); electron microscopy; mechanical properties.

\section{Introduction}

Graphene, a two-dimensional (2D) monolayer of carbon atoms arranged in honeycomb lattice (Geim and Novoselov 2007), shows great potential to fabricate nanocomposites with high performances and novel functionalities for a range of applications such as electric conductive composites (Vuluga et al 2011), supercapacitors (Fan et al 2010; Chen et al 2011), sensors (Stine et al 2010; An et al 2011), biomaterials (Park et al 2010), batteries (Liang and Zhi 2009), ultrafast laser mode-locker (Zhang et al 2009; Sun et al 2010), and thermally stable and mechanically reinforced materials. This is particularly important in the manufacture of high performance materials to obtain a uniform or homogeneous distribution of graphene sheets in various matrices (Allen et al 2010; Loh et al 2010; Cravotto and Cintas 2010). Similar to CNTs, pristine graphene sheets are prone to congregate in composites unfortunately for their strong $\pi-\pi$ stacking between layers and incompatible surface characteristics with the polymer matrices, posing a big obstacle for its application in nanocomposites. Therefore, chemical functionalization is needed as well to achieve the singlesheet dispersion of graphene in far-ranging polymer composites with optimum performances (Xu and Gao 2010). So far, two major approaches, viz. non-covalent (Liu et al 2010a; Lee et al 2011) and covalent (Pham et al 2010), have been developed to synthesize graphene/polymer composites. Davis et al (Liu et al 2010b) have successfully synthesized $\mathrm{pH}$ - and thermosensitive graphene-polymer composites by

\footnotetext{
*Author for correspondence (polymer_jiang@ hotmail.com)
}

attaching pyrene-terminated poly $(N$-isopropylacrylamide $)$ (PNIPAAm) and onto the basal plane of graphene sheets via $\pi-\pi$ stacking. However, the non-covalent approach is insufficient for practical applications. Hence, covalent approaches draw more attention and have many advantages over the non-covalent one. Among them, coupling of the polymer on graphite oxide sheets to form composites, viz. "grafting to" method, is the most common approach in practice (Pramoda et al 2010; Deng et al 2011). Herein, we report a new approach to prepare hyperbranched polymer/graphene nanocomposites from graphite oxide via in situ grafting method.

\section{Experimental}

\subsection{Materials}

Graphite powder $(40 \mu \mathrm{m})$ was obtained from Qingdao Henglide Graphite Co., Ltd. Concentrated sulfuric acid $\left(\mathrm{H}_{2} \mathrm{SO}_{4}, 98 \%\right)$, potassium permanganate $\left(\mathrm{KMnO}_{4}\right)$, sodium nitrate $\left(\mathrm{NaNO}_{3}\right)$ and methylene blue were purchased from Shanghai Reagents Company and used as received. 3-Ethyl3 -oxetanethanol was synthesized according to the literature procedures (Jiang et al 2005, 2006).

\subsection{Preparation of hyperbranched polymers-graphene (HPG) composites}

First, graphite oxide (GO) was synthesized through graphite powder oxidation with sulfuric acid and potassium permanganate $\left(\mathrm{H}_{2} \mathrm{SO}_{4}-\mathrm{KMnO}_{4}\right)$ (Hummers and Offerman 1958). 
The chemical grafting of hyperbranched polymer onto the graphite oxide sheets was carried out by the "grafting from" approach based on in situ ring-opening polymerization of 3-ethyl-3-oxetanethanol (Jiang et al 2005). In a typical experiment, $100 \mathrm{mg}$ of graphite oxide and $10 \mathrm{ml} \mathrm{CHCl}_{3}$ were added into a Schleich tube to form a dispersion solution under sonication at room temperature for $1 \mathrm{~h}$. And then, $5 \mathrm{ml}$ of the monomer, 3-ethyl-3-oxetanethanol, and catalyst were added into the reaction tube. After reaction for desired time, a homogeneous black dispersion was obtained. The original dispersion was dialyzed against $\mathrm{CHCl}_{3}$ for $24 \mathrm{~h}$ to remove the un-covalented polymer to yield a stable black supernatant. The stable black supernatant was collected and freeze-dried to obtain hyperbranched polymers (HPs)/graphene nanocomposites which are designed as HPG-1 (polymerization at $50^{\circ} \mathrm{C}$ ) and HPG-2 (polymerization at $0^{\circ} \mathrm{C}$ ).

\subsection{Characterization}

The ${ }^{1} \mathrm{H}$ NMR spectra were recorded on an AVANCE AV $400 \mathrm{MHz}$ Digital FT-NMR spectrometer operating at $400 \mathrm{MHz}$ using deuterated DMSO-d6 as a solvent. The sizes and morphologies of the resultant samples were characterized by JSM-2100 transmission electron microscopy (TEM) at an accelerating voltage of $200 \mathrm{kV}$, whereby a small drop of sample solution was deposited onto a carbon-coated copper EM grid (200 mesh) and dried at room temperature at atmospheric pressure. ULTRA-55 field-emission scanning electron microscopy (FE-SEM) was also applied to investigate sizes and morphologies of the resultant samples. Thermogravimetric analysis (TGA) was performed on a Pyris Diamond 1 instrument (America) at a heating rate of $20^{\circ} \mathrm{C}$ $\mathrm{min}^{-1}$ from $25-550{ }^{\circ} \mathrm{C}$ in a flow of nitrogen. Fourier trans- form infrared (FT-IR) spectra were recorded on a Nicolet 5700 spectrophotometer using $\mathrm{KBr}$ pellets for samples. The mechanical properties of nanocomposites were measured using an universal testing machine (CMT-4102, SANS Group, China) at room temperature. A load cell of $500 \mathrm{~N}$ was employed and the tensile rate imposed was $1 \mathrm{~mm} / \mathrm{min}$. All samples were cut into a dumbbell shape with a razor blade. More than five tests were conducted for each sample, from which the mean values and standard deviations were derived.

\section{Results and discussion}

In our study, graphite oxide was synthesized by the modified Hummer's and Offeman's method (Hummers and Offerman 1958). Subsequently, the chemical grafting of hyperbranched polymer onto the graphite oxide sheets was carried out by the "grafting from" approach based on in situ ring-opening polymerization of 3-ethyl-3-oxetanethanol (Jiang et al 2005, 2006). The synthesis of HPGs is schematically shown in scheme 1. The first step is to synthesize GO sheets by completely oxidizing natural graphite with the aid of ultrasonication. Scanning electron microscopy (SEM) and transmission electron microscopy (TEM) images of the GO sheets show that the graphene oxide are slightly wrinkled and folded, and their sizes are larger than $1 \mu \mathrm{m}$ (figure S1, see Electronic Supplementary Material). The FT-IR spectra of GO and natural graphite (figure S2, see Electronic Supplementary Material) show that the characteristic peaks of the GO include the $\mathrm{C}=\mathrm{O}$ carbonyl stretching vibration at $1720 \mathrm{~cm}^{-1}$, the $-\mathrm{O}-\mathrm{H}$ deformation vibration at $1400 \mathrm{~cm}^{-1}$, the $-\mathrm{OH}$ stretching at $1230 \mathrm{~cm}^{-1}$ and the $\mathrm{C}-\mathrm{O}$ stretching at $1060 \mathrm{~cm}^{-1}$. The resultant GO can be easily dissolved in water without dispersants, as shown in figure 1B. The $\mathrm{X}$-ray diffraction (XRD) pattern

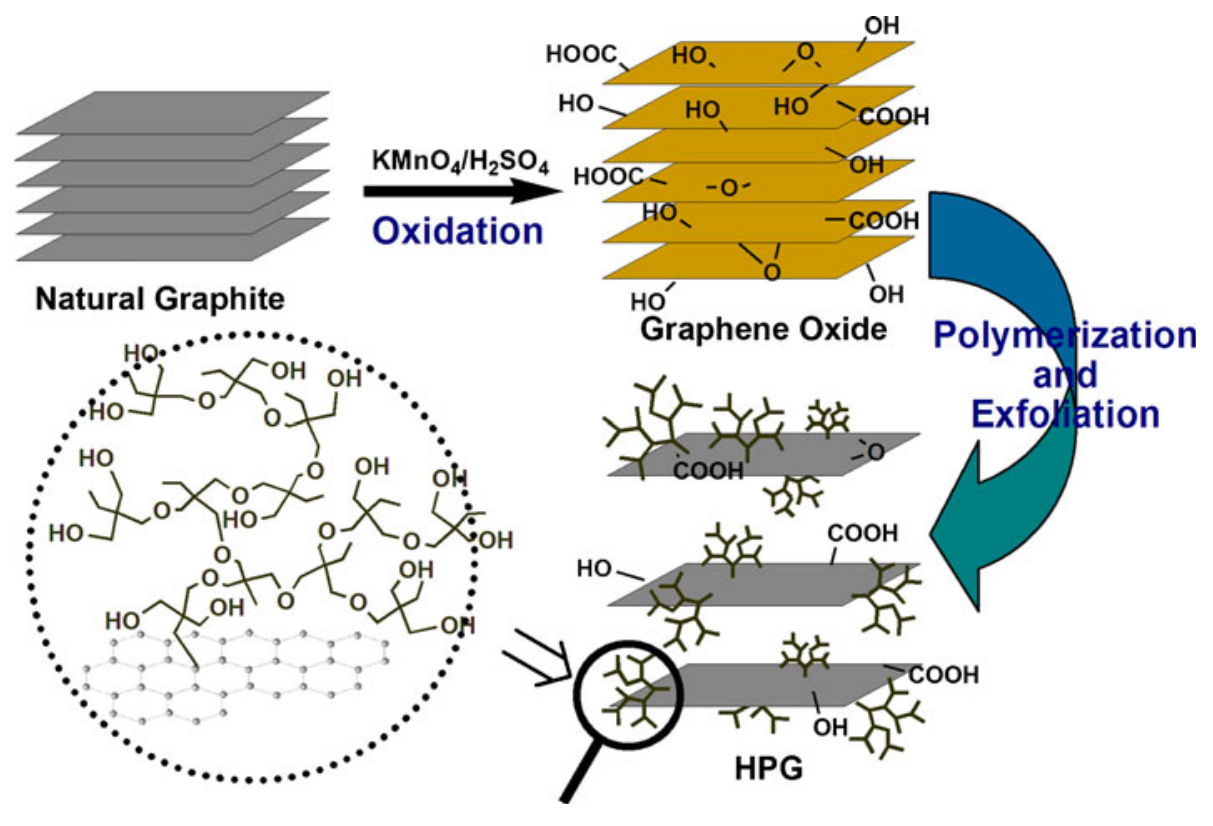

Scheme 1. Schematic of synthesis of hyperbranched polymers (HPs) on graphene sheets. 


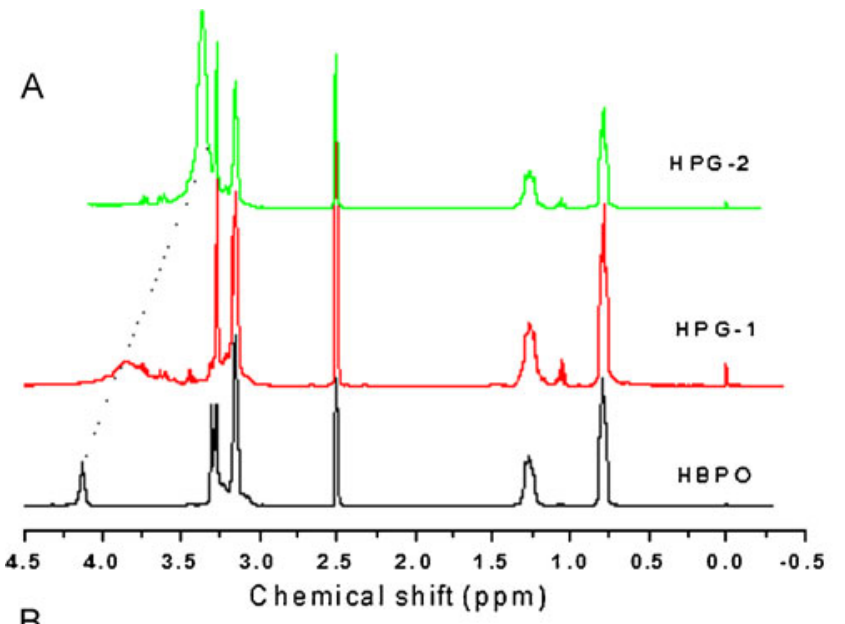

B

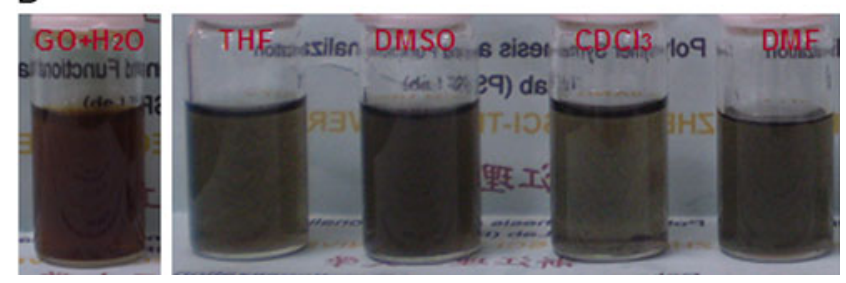

Figure 1. ${ }^{1} \mathrm{H}$ NMR spectra of pure $\mathrm{HPs}$ and $\mathrm{HPG}$ in $\mathrm{CDCl}_{3}$ (A) and solubility of GO in $\mathrm{H}_{2} \mathrm{O}$ and $\mathrm{HPG}$ in different organic solvents $(\mathbf{B})$.

of GO and natural graphite further confirms the efficient oxidation of graphite (figure S3, see Electronic Supplementary Material).

HPGs were synthesized by cationic polymerization of 3-methyl-3-oxetanemethanol directly initiated by $\mathrm{BF}_{3} \cdot \mathrm{Et}_{2} \mathrm{O}$ according to the literature procedures (Jiang et al 2006). After successful grafting, the covalently grafted chains of HPs change the surface properties of graphene sheets. The resultant composites exhibit good solubility in the solvents of polymers. To affirm the successful grafting effect on the graphene sheets, we took ${ }^{1} \mathrm{H}$ NMR measurement; the protons from HPs can be observed as shown in figure $1 \mathrm{~A}$. The ${ }^{1} \mathrm{H}$ NMR spectra of HPGs were identical to that of the homopolymer of 3-methyl-3-oxetanemethanol (HPs) obtained at $-10^{\circ} \mathrm{C}$. A comparison of the ${ }^{1} \mathrm{H}$ NMR spectra of HPG-1, HPG-2 and HPs reveals that the peak at $4.20 \mathrm{ppm}$ in HPs, which can be assigned to $-\mathrm{CH}_{2}$ connected to oxygen atoms, transfer to $3.80 \mathrm{ppm}$ in HPG-1 and $3.50 \mathrm{ppm}$ in HPG-2 for the increasing degree of branching at higher polymerization temperature (Zhu et al 2009). The resultant HPGs are dissolved homogenously in DMF, THF, $\mathrm{CHCl}_{3}$ and DMF, without aggregation of graphene sheets for half a month, implying the high efficiency of polymer grafting, as shown in figure $1 \mathrm{~B}$.

The morphological properties of GO and HPGs were studied by atomic force microscopy (AFM). AFM is commonly used to study the surface morphology of a material in nanometer scale. It provides high-resolution imaging and measurement of surface topography and properties on the molecular scale. The samples for AFM were deposited by spin-casting from its THF solution. Figures $2 \mathrm{~A}$ and $\mathrm{C}$ show the tapping mode AFM images of GO and HPG sheets, respectively. The grafted chains on two sides of graphene sheets heighten its thickness to about $12 \mathrm{~nm}$, compared to the thickness of $\mathrm{GO}$ of about $0.8 \mathrm{~nm}$, which is proved by Gao's group (Xu and Gao 2010). In the case of HPGs, the grafted polymers cover the whole plane of graphene with bump surface (figure 2D), compared to the even height of GO (figure 2B), suggesting the high density of grafting HPs with a fabulous efficiency. Interestingly, irregularities of the HPGs' surface represent different sized HPs molecules nonuniformly anchored on the substrate surface for the functional initiator sites' nonuniform distribution.

In order to further investigate the morphologies of the prepared hybrids, HR-TEM images were recorded. Figures 2E and $F$ show TEM images of GO and HPG. From the TEM image of GO, the presence of graphene layer with smooth surface can be seen. After grafting of HP onto the surface of GO, a significant change was observed. Figure $2 \mathrm{~F}$ shows morphology of HPG, in which the dark regions are related to the grafted HPs onto the GO surface. It is interesting to note that the intensity of dark colour on the basal plane of GO is relatively higher than edges, indicating higher density of grafted HPs on these regions. It can be explained by higher density of hydroxyl functional groups on the basal plane of GO (Stankovich et al 2007). After HPs were grafted onto $\mathrm{GO}$, the strong peak of GO in XRD at $11.7^{\circ}$ disappeared, further indicating that $\mathrm{PG}$ was successfully grafted onto the GO surface (figure S3, see Electronic Supplementary Material).

By contrast with the FT-IR curve of GO, the obvious decrease of the oxygenated functional groups on the sheets in HPGs may be due to the small content of graphene sheets in the composites (figure S3, see Electronic Supplementary Material). In figure 3, TGA traces of pristine graphite shows a negligible weight loss, while, GO shows much lower thermal stability. The major weight loss of GO occurs at $180^{\circ} \mathrm{C}$, which can be assigned to the pyrolysis of the labile oxygencontaining functional groups, yielding $\mathrm{CO}, \mathrm{CO}_{2}$ and steam (Stankovich et al 2007). However, HPGs are more stable than HPs. The increased thermal stability of the hybrid nanostructures could be attributed to the chemical grafting of HPs onto the GO surface.

Given the excellent elastic modulus and intrinsic strength of graphene sheets ( $\mathrm{Li}$ and Kaner 2008; Lee et al 2008; Park and Ruoff 2009; Geim 2009), we examined the effect of functionalized graphene sheets on the mechanical properties of HPGs films. Figure 4A presents the representative stress strain curves of the pristine HPs and HPGs films. Pure HPs perform a tensile strength of $12.5 \mathrm{MPa}$ and a break elongation of $140 \%$. At low branching degree of HPs, HPG-1 shows distinct yield points for crystalline polymer, probably for the orientation-induced crystallization at high draw ratio. HPG-1 has a tensile strength of $22 \mathrm{MPa}$, which is higher than that of HPs by $76 \%$, and performs a lower break elongation of $120 \%$. At higher branching degree of HPs, tensile strength of HPG-2 enhanced up to $27 \mathrm{MPa}(2 \cdot 2$ folds 

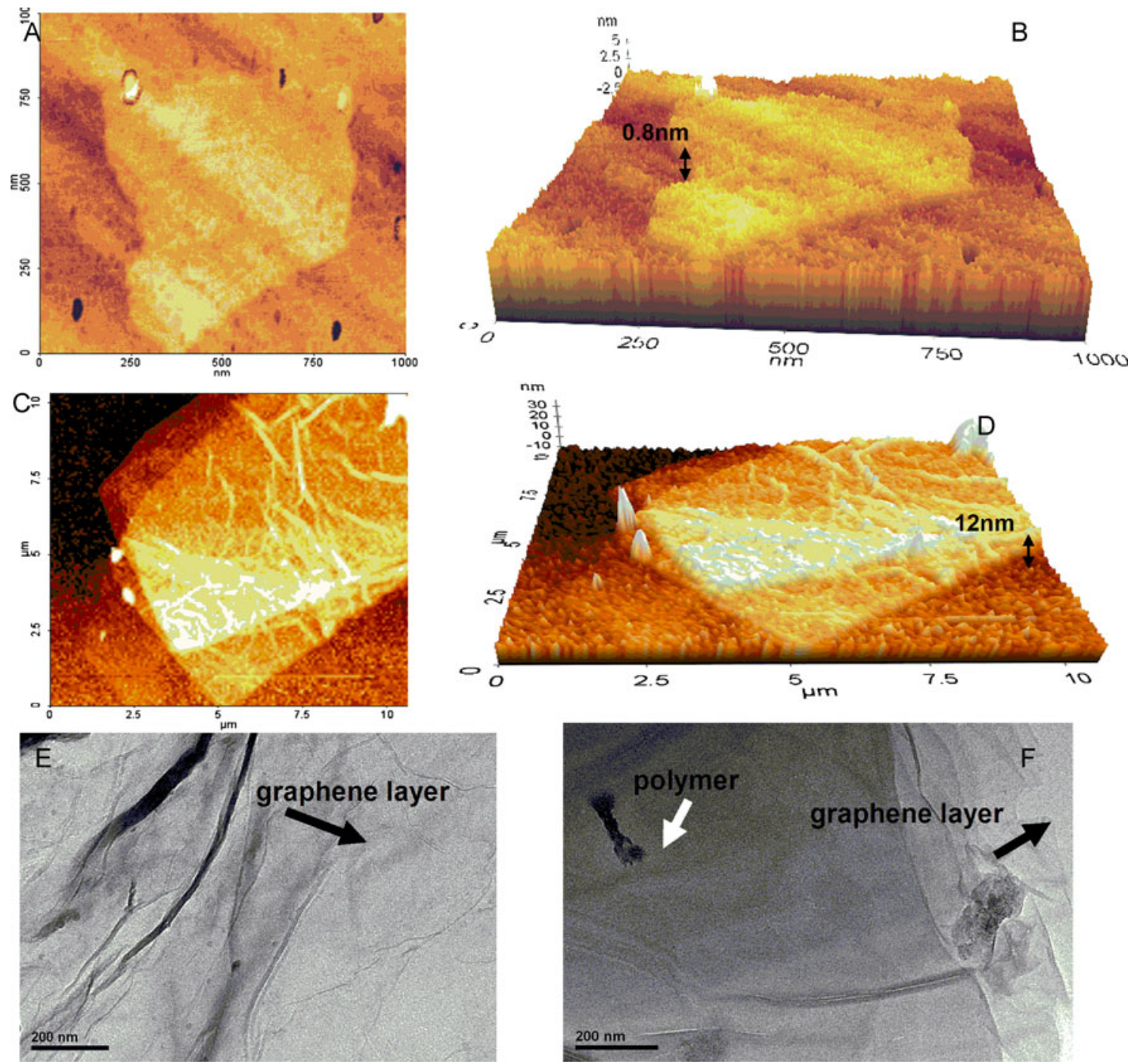

Figure 2. AFM height image of GO (A) and HPGs (D) on mica deposited from $\mathrm{CHCl}_{3}$ solution. 3D-view images of $\mathrm{GO}(\mathbf{B})$ and HPGs (E) on mica spinning-cast from solution of $\mathrm{CHCl}_{3}$. TEM images of $\mathrm{GO}(\mathbf{C})$ and HPGs $(\mathbf{F})$.

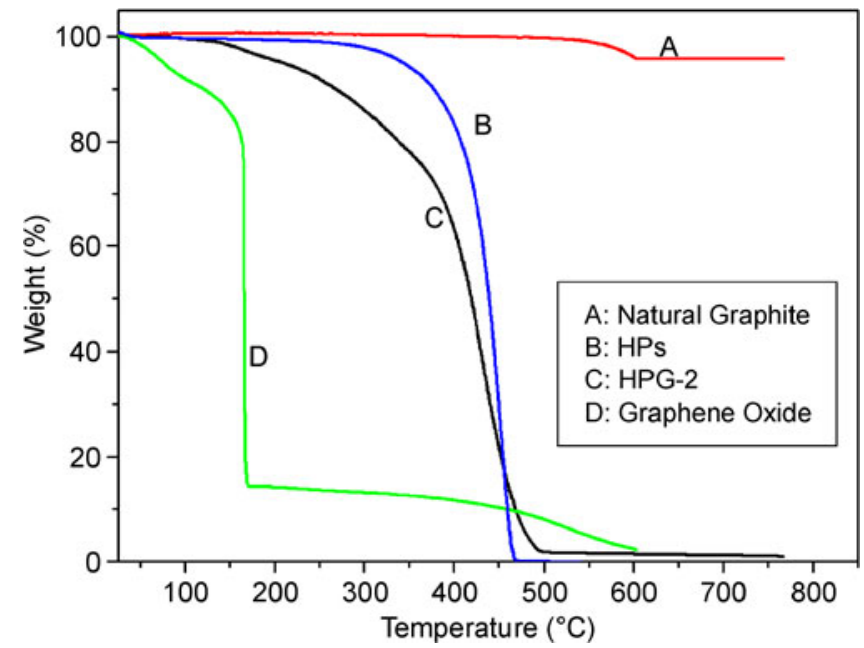

Figure 3. TG curves of pure natural graphite, HPs, HPG-2 and graphene oxide. over that of HPs), accompanying with further decreasing of break elongation at $110 \%$. HPG-2 has a higher degree of branching and thus stronger intermolecular forces and tensile strength. Undoubtedly, excellent reinforcement of graphene could be attributed to the good dispersion of graphene sheets in composites and the strong interaction between the HPsgrafted graphene and HPs matrix. Figure 4B shows photograph of the as-made composites after being removed from the filter membrane. The as-made composite is very flexible and shows certain mechanical robustness when subjected to bending.

This result has been proved in some studies. Wang et al (2011a) reported that reinforcement of graphene resulted in the increase of up to 27.0 and $92.8 \%$ in the tensile strength and Young's modulus of the nanocomposites, respectively, compared to neat LDPE. Compared to pure poly $\left[2,2^{\prime}-\right.$ ( $p$-oxydiphenylene)-5,5'-bibenzimidazole] (OPBI), the composites showed a $17 \%$ increase in Young's modulus, $33 \%$ 


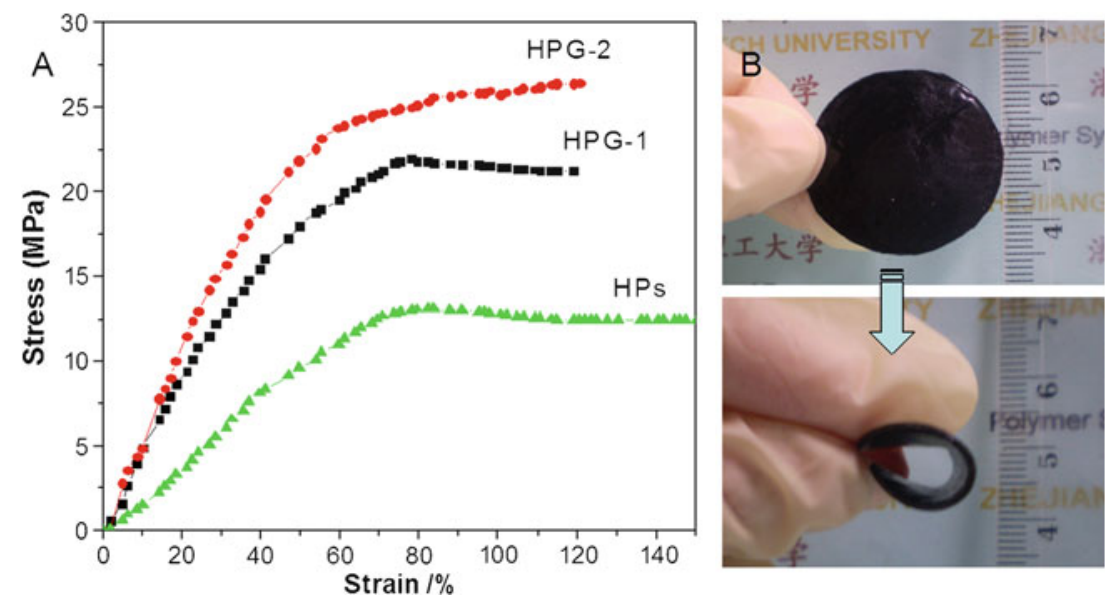

Figure 4. Stress-strain curves of neat HPs and HPG composites (A) and as-prepared composites show mechanical robustness when subjected to bending (B).

increase in tensile strength and $88 \%$ improvement in toughness by the addition of only $0.3 \mathrm{wt} . \%$ of GO (Wang et al $2011 b)$. In the case of graphene/PVA composites, a $150 \%$ improvement of tensile strength and a nearly 10 times increase of Young's modulus are achieved at a graphene loading of $1.8 \mathrm{vol} \%$ (Zhao et al 2010). The mechanism behind the enhancement of the mechanical and thermal properties of nanocomposites was attributed to the strong interaction between the HPG molecules and GO. As the graphene monolayers were dispersed on the molecular level in the HPG matrix, although the content of graphene was very low, a large number of graphene monolayers were still incorporated into the HPG matrix (Ren et al 2011). It is speculated that in the HPG nanocomposites, GO could act as anchoring sites for linking polymer chain due to the strong interaction between polymer and GO. Therefore, the stress can be efficiently transferred from matrix to GO.

\section{Conclusions}

In summary, an efficient strategy for the preparation of organic-inorganic hybrid material containing graphene oxide and HPs by an in situ grafting approach is demonstrated. The resultant nanocomposites show good solubility in the solvents of polymers. The efficient HPs grafting makes the graphene homogeneously dispersed in polymer matrix and increased the mechanical properties of resultant composites. The in situ grafting polymerization approach paved the way to prepare graphene-based nanocomposites with high performances and novel functionalities.

\section{Electronic supplementary material}

Supplementary material pertaining to this article is available on the Bulletin of Materials Science website (www.ias.ac.in/matersci).

\section{Acknowledgements}

This work was financially supported by the Qianjiang Talents Project of Zhejiang Province (2010R10023), the Scientific Research Foundation for the Returned Overseas Chinese Scholars, the State Education Ministry (1001603C), the Natural Science Foundation of Zhejiang Province (Y4100045), the Key Bidding Project of Zhejiang Provincal Key Lab of Fiber Materials and Manufacturing Technology, Zhejiang Sci-Tech University(S2010002) and the Program for Changjiang Scholars and Innovative Research Team in University (PCSIRT: 0654).

\section{References}

Allen M J, Tung V C and Kaner R B 2010 Chem. Rev. 110132

An X, Butler T W, Washington M, Nayak S K and Kar S 2011 ACS Nano 51003

Chen Y, Zhang X, Zhang D, Yu P and Ma Y 2011 Carbon 49 573

Cravotto G and Cintas P 2010 Chem. Eur. J. 165246

Deng Y, Li Y, Dai J, Lang M and Huang X 2011 J. Polym. Sci. Part A, Polym. Chem. 491582

Fan Z, Yan J, Zhi L, Zhang Q, Wei T, Feng J, Zhang M, Qian W and Wei F 2010 Adv. Mater. 223723

Geim A K 2009 Science 3241530

Geim A K and Novoselov K S 2007 Nat. Mater. 6183

Hummers W and Offerman R 1958 J. Am. Chem. Soc. 801339

Jiang G, Wang L, Chen T, Yu H, Wang C and Chen C 2005 Polymer 465351

Jiang G, Wang L and Chen W 2006 Eur. Polym. J. 423333

Lee C G, Wei X D, Kysar J W and Hone J 2008 Science 321 385

Lee D Y, Khatun Z, Lee J H, Lee Y K and In I 2011 Biomacromolecules 12336

Li D and Kaner R B 2008 Science 3201170

Liang M H and Zhi L J 2009 J. Mater. Chem. 195871 
Liu J, Yang W, Tao L, Li D, Boyer C and Davis T P 2010a J. Polym. Sci. Part A, Polym. Chem. $\mathbf{4 8} 425$

Liu J, Tao L, Yang W, Li D, Boyer C, Wuhrer R, Braet F and Davis T P 2010b Langmuir 2610068

Loh K P, Bao Q, Ang P K and Yang J 2010 J. Mater. Chem. 202277

Park S and Ruoff R S 2009 Nat. Nanotechnol. 4217

Park S, Mohanty N, Suk J W, Nagaraja A, An J, Piner R D, Cai W W, Dreyer D R, Berry V and Ruoff R S 2010 Adv. Mater. 22 1736

Pham T A, Kumar N A and Jeong Y T 2010 Syn. Mater. 160 2028

Pramoda K P, Hussain H, Koh H M, Tan H R and He C B 2010 J. Polym. Sci. Part A, Polym. Chem. 484262

Ren P-G, Yan D-X, Chen T, Zeng B-Q and Li Z-M 2011 J. Appl. Polym. Sci. 1213167

Stankovich S, Dikin D A, Piner R D, Kohlhaas K A, Kleinhamma A, Jia Y, Wu Y, Nguyen S T and Ruoff R S 2007 Carbon 451558
Stine R, Robinson J T, Sheehan P E and Tamanaha C R 2010 Adv. Mater. 225297

Sun Z P, Hasan T, Torrisi F, Popa D, Privitera G, Wang F Q, Bonaccorso F, Basko D M and Ferrari A C 2010 ACS Nano 4803

Vuluga D, Thomassin J M, Molenberg I, Huynen I, Gilbert B, Jérôme C, Alexandre M and Detrembleur C 2011 Chem. Commun. 472544

Wang J, Xu C, Hu H, Wan L, Chen R, Zheng H, Liu F, Zhang M, Shang X and Wang X 2011a J. Nanopart. Res. 13869

Wang Y, Shi Z, Fang J, Xu H and Yin J 2011b Carbon 491199

$\mathrm{Xu} \mathrm{Z}$ and Gao C 2010 Macromolecules 436716

Zhang H, Bao Q L, Tang D Y, Zhao L M and Loh K P 2009 Appl. Phys. Lett. 95141103

Zhao X, Zhang Q H, Chen D J and Lu P 2010 Macromolecules 43 2357

Zhu Q, Wu J, Tu C, Shi Y, He L, Wang R, Zhu X and Yan D 2009 J. Phys. Chem. B113 5777 